\title{
HABILIDADES DE ORDENAÇÃO E RESOLUÇÃO TEMPORAL EM CRIANÇAS COM DESVIO FONOLÓGICO
}

\author{
Ordering abilities and temporal resolution \\ in children with phonological disorders
}

\author{
Júlia Larissa Ferreira dos Santos ${ }^{(1)}$, Letícia Maria Martins Vasconcelos Parreira ${ }^{(2)}$, \\ Rita de Cássia Duarte Leite ${ }^{(3)}$
}

\begin{abstract}
RESUMO
Objetivo: verificar o desempenho de crianças com transtornos fonológicos em testes de ordenação e resolução temporal. Métodos: a amostra foi composta por 12 indivíduos, de ambos os gêneros, com idade entre 5 e 13 anos, com diagnóstico de desvio fonológico. Os indivíduos foram submetidos à anamnese, meatoscopia, audiometria tonal via aérea e óssea, PCC (porcentagem de consoantes corretas), e o RGDT (teste de detecção de gaps) e o PPS (teste de padrão de sequência). Resultados: em relação ao grau de severidade, verificou-se que $67 \%$ apresentaram desvio fonológico médio e $33 \%$ médio-moderado. Em relação aos testes RGDT e PPS, os sujeitos apresentaram respostas dentro dos padrões normativos estabelecidos pela literatura, em ambos os testes. Na comparação entre o desempenho no teste PPS e o grau de severidade, observou-se que quanto maior o grau de severidade, pior o desempenho do indivíduo no teste, o mesmo não foi observado em relação ao RGDT. Conclusões: a amostra estudada apresentou desempenho adequado nos testes de processamento temporal. Entretanto, os resultados evidenciaram que existe uma relação estatisticamente significante entre a severidade do desvio e o desempenho no teste PPS, demonstrando a influência da severidade do desvio no desempenho dos indivíduos, considerando a habilidade de ordenação temporal.
\end{abstract}

DESCRITORES: Fala; Percepção Auditiva; Audição

\section{INTRODUÇÃO}

Parte da tarefa de aquisição de uma determinada língua engloba o aprendizado de quais são os sons usados e como eles se organizam, para a formação do sistema fonológico alvo. A maioria das crianças executa essa tarefa sem dificuldades e, aproximadamente, aos cinco anos de idade, já pro-

(1) Fonoaudióloga clínica da Prefeitura Municipal de Biquinhas, MG, Brasil; Pós-graduanda em Audiologia na FEAD

- Centro de Gestão Empreendedora.

(2) Fonoaudióloga; Professora do curso de Graduação em Fonoaudiologia da FEAD - Centro de Gestão Empreendedora, Belo Horizonte, MG, Brasil; e da Universidade FUMEC, Belo Horizonte, MG, Brasil; Mestre em Ciências da Saúde pela Universidade Federal de Minas Gerais.

(3) Fonoaudióloga; Professora do curso de Graduação em Fonoaudiologia da FEAD - Centro de Gestão Empreendedora, Belo Horizonte, MG, Brasil; Mestre em Ciências da Saúde pela Universidade Federal de Minas Gerais.

Conflito de interesses: inexistente duz os sons adequadamente e apenas nas sequências permitidas ${ }^{1}$.

Nos desvios fonológicos, o processamento dessas informações fonológicas, acontece de maneira diferente do esperado, estando além de uma dificuldade apenas articulatória ${ }^{2}$. Caracterizando, assim, uma inadequação no estabelecimento adequado do sistema fonológico alvo e do sinal de entrada auditivo recebido ${ }^{3}$.

Para atingir o reconhecimento e compreensão dos sons da fala, fator extremamente importante para o desenvolvimento da língua oral, torna-se necessário um adequado processamento do input da língua falada por parte da criança. Portanto, muitos problemas no desenvolvimento dos sons da fala têm sido relacionados a dificuldades/desordens no processamento auditivo ${ }^{4}$.

O processamento auditivo (PA) é definido como uma série de processos que ocorrem no tempo, permitindo assim que um indivíduo realize análise acústica e metacognitiva dos sons, 
relacionando-se as habilidades envolvidas na decodificação, na organização e na codificação da informação sensorial auditiva, levando a percepção e compreensão da informação sonora pelo indivíduo ${ }^{4}$. Estes processos são aplicáveis a estímulos verbais e não-verbais podendo afetar diversas áreas incluindo a fala e a linguagem ${ }^{5}$.

As funções do sistema nervoso auditivo central são influenciadas pela sequência de eventos sonoros que ocorrem no tempo, ou seja, pelo processamento da informação temporal ${ }^{6}$. O processamento temporal refere-se à análise das informações temporais do sinal auditivo, como a percepção de um determinado som ou sua diferenciação dentro de um domínio de tempo restrito ou definido. Sendo assim, é considerada uma habilidade fundamental na percepção auditiva de sons verbais e nãoverbais, na percepção de música, ritmo e pontuação, na discriminação de pitch, de duração e de fonemas ${ }^{7,8}$.

Dentre as habilidades do processamento temporal, encontra-se a ordenação e resolução temporal. Tais habilidades são bastante investigadas, devido a sua importância na aquisição da fala, estando diretamente relacionadas à percepção e discriminação fonêmica necessárias à formação do sistema fonológico da língua alvo ${ }^{7}$.

A ordenação ou sequencialização temporal consiste em uma habilidade auditiva, que envolve a percepção e o processamento de dois ou mais estímulos auditivos em sua ordem de ocorrência no tempo ${ }^{6,7}$. A resolução temporal é a habilidade responsável pela detecção de intervalos de tempo entre estímulos sonoros, de mudanças rápidas e bruscas no estimulo sonoro, ou detectar o menor intervalo de tempo necessário para que um indivíduo possa perceber diferenças entre sinais sonoros ${ }^{7,9}$.

Alterações nessas habilidades temporais geram dificuldades na percepção de estímulos que se modificam rapidamente e isso pode afetar a discriminação e o processamento fonológico dos sons da língua, interferindo na compreensão de fala e, consequentemente, na aquisição do sistema fonológico alvo ${ }^{10,11}$. Diante desta relação entre o processamento temporal e as habilidades fonológicas inerentes à aquisição sem desvios da linguagem oral, verifica-se a necessidade de identificação de alterações no processamento temporal de crianças com desvios fonológicos.

Este estudo tem por objetivo verificar o desempenho de crianças com desvio fonológico nos testes de ordenação e resolução temporal.

\section{MÉTODOS}

Trata-se de um estudo transversal do tipo descritivo, realizado na Clínica escola de Fonoaudiologia da FEAD, localizada na cidade de Belo
Horizonte - MG. Os participantes deste estudo foram crianças de ambos os gêneros e que obedeciam aos seguintes critérios: diagnóstico fonoaudiológico de desvio fonológico; idade superior a 5 anos; concordância com a participação do estudo; acuidade auditiva normal (limiares auditivos até de $15 \mathrm{dBNA})^{12}$. Foram excluídas as crianças que apresentavam: alterações visuais, neurológicas e no desenvolvimento neuropsicomotor; história clínica atual ou pregressa de alteração otológica; ter realizado qualquer tipo de treinamento musical.

Após a composição da amostra, foi realizada uma anamnese com responsável por cada participante. Posteriormente, os indivíduos foram submetidos à meatoscopia, audiometria tonal liminar via aérea em todas as frequências e via óssea para descartar presença de gap aéreo-ósseo e logoaudiometria, realizados em audiômetro de dois canais, modelo AC33, de marca Interacoustics, fone TDH39 e vibrador B71.

Para a classificação do grau de severidade do desvio fonológico dos participantes, foi utilizado o método Porcentagem de Consoantes Corretas (PCC) ${ }^{13}$. A porcentagem obtida forneceria o grau do desvio fonológico.

Em seguida, os indivíduos foram submetidos aos dois testes de processamento temporal selecionados: RGDT e PPS. Estes testes foram apresentados a $50 \mathrm{dBNS}$, tendo como padrão a média tritonal (500, 1000 e $2000 \mathrm{~Hz}$ ), de cada indivíduo.

O Random Gap Detection Test (RGDT) constitui-se de um teste que avalia habilidade de resolução temporal ${ }^{14}$. Consiste em uma apresentação de uma sequência de nove estímulos, com intervalo de tempo, em que os participantes são instruídos a responder verbalmente se está escutando um ou dois sons. Trata-se de um teste diótico, em que os estímulos são apresentados nas frequências de $500,1000,2000,4000 \mathrm{~Hz}$, com intervalos de tempo de 0 a 40 milisegundos aleatórios.

O PPS (Picth Patern Sequency) é um teste que verifica a habilidade de ordenação temporal, possui duas versões, a infantil e a adulta, as quais diferem nos aspectos de duração do tom e intervalo entre os estímulos. Este teste diótico foi apresentado monoauralmente, em ambas as versões. Nas crianças de até 09 anos de idade foi realizada a versão infantil, utilizando estímulos contendo três tons apresentando as frequências agudas $(1430 \mathrm{~Hz})$ ou graves $(880 \mathrm{~Hz})$. Para crianças com idade acima de 09 anos, foi utilizada a versão adulta, nas mesmas frequências e ordem de apresentação aleatória. Cada participante foi instruído a repetir os itens na forma de imitação, por meio de murmúrio, e/ou nomeação, com as terminologias de fino e grosso. 
Este trabalho foi aprovado pelo Comitê de Ética em Pesquisa em seres humanos de FEAD.

A análise descritiva foi realizada por meio das medidas de tendência central e medidas de dispersão. Para comparação, foi utilizada análise inferencial por meio do teste Exato de Fischer, T de Student pareado e não pareado, ambos com nível de significância de $5 \%$ (valor $p \leq 0,05$ ).

\section{RESULTADOS}

Participaram deste estudo 12 crianças de ambos os gêneros, sendo $75 \%$ (8) do gênero masculino e $25 \%$ (4) feminino, com média de idade de 8 anos e 5 meses ( $\pm 2,1$ anos). Considerando-se o grau de severidade dos desvios fonológicos observou-se que $67 \%$ (8) apresentaram desvio fonológico médio e 33\% (4) médio-moderado.

$\mathrm{Na}$ Tabela 1, observam-se os resultados dos limiares de detecção de gap obtidos no RGDT, por frequência e o gap total obtido pelos participantes.

Nas 12 crianças avaliadas, encontrou-se resposta adequada do PPS (nomeando) para nove
(75\%) crianças com uma média de acertos de $82 \%$ quando os estímulos foram apresentados a OD e $80 \%$ quando foram apresentados a OE e resposta alterada para três crianças (25\%). Portanto, os três indivíduos necessitaram da realização do teste na versão imitando. Pôde-se observar que destes indivíduos, dois apresentaram respostas dentro da normalidade e apenas um indivíduo com resultado alterado em ambas as modalidades, demonstrando alteração na habilidade de ordenação temporal de frequências. Não foi encontrada diferença estatisticamente significante entre as orelhas direita e esquerda no PPS modalidade imitando e nomeando.

A comparação entre o limiar de detecção de gap dos indivíduos no teste RGDT e o grau de severidade dos desvios fonológicos, pode ser observada na Tabela 2.

A comparação entre as variáveis, grau de severidade do desvio fonológico e o resultado obtido pelos indivíduos no teste PPS, pode ser observada na Tabela 3, verificando-se uma relação estatisticamente significante $(p<0,05)$, apesar do pequeno de número de participantes da pesquisa.

Tabela 1 - Respostas obtidas no RGDT por frequência

\begin{tabular}{lccccc}
\hline \multirow{2}{*}{ Valores descritivos } & \multicolumn{5}{c}{ Frequências $\mathbf{( H z )}$} \\
\cline { 2 - 6 } Média & $\mathbf{5 0 0}$ & $\mathbf{1 0 0 0}$ & $\mathbf{2 0 0 0}$ & $\mathbf{4 0 0 0}$ & Res. Final - ms \\
\cline { 2 - 6 } Desvio padrão & 14,16 & 14,78 & 15,41 & 15,83 & 15 \\
Mediana & 3,5 & 7,2 & 5,4 & 5,9 & 4,4 \\
Mínimo & 15 & 15 & 15 & 15 & 15 \\
Maximo & 5 & 5 & 10 & 5 & 7,5 \\
\hline
\end{tabular}

Tabela 2 - Comparação entre limiar de detecção de gap e o grau de severidade do D.F. da amostra estudada

\begin{tabular}{cccc}
\hline & \multicolumn{4}{c}{ Grau de Severidade } \\
\hline RGDT - limiar de gap & Médio & Médio-moderado \\
Média & 16,12 & 13,43 & \\
Desvio padrão & 5,99 & 1,19 & $\mathrm{p}=0,58$ \\
\hline
\end{tabular}

Teste T de Student

Tabela 3 - Correlação entre o grau de severidade do desvio fonológico e o resultado do PPS na amostra estudada

\begin{tabular}{ccccc}
\hline & Teste PPS \\
\hline Grau de severidade & Normal & Alterado & Total \\
Médio & 3 & 0 & 8 & \\
Médio-moderado & 1 & 3 & 4 & $\mathbf{p}=\mathbf{0 , 0 1 1}^{*}$ \\
Total & 9 & 3 & 12 & \\
\hline
\end{tabular}

*relação estatisticamente significante. Teste Exato de Fischer 


\section{DISCUSSÃO}

Alterações na expressão e recepção de linguagem podem ser decorrentes de qualquer prejuízo na habilidade de ouvir e compreender a informação sonora, que depende da capacidade inata do indivíduo e das experiências deste com o meio. Essa percepção auditiva depende, também, de estímulos constantes e progressivos que favorecem a compreensão sonora verbal/não-verbal e das informações supra-segmentais presentes na fala, estando diretamente relacionada aos aspectos temporais auditivos. Um déficit em qualquer um desses aspectos, tanto na ordenação quanto na resolução temporal, pode alterar o sistema fonológico e, consequentemente haverá problemas de linguagem oral ${ }^{15}$.

Ao analisar a habilidade de resolução temporal nas crianças com transtorno fonológico, verificou-se a presença de limiares de detecção de gap dentro da normalidade, concordando com estudo realizado por Feniman, Keith e Cunnigham ${ }^{16}$ em que os autores, ao analisar 17 crianças com alterações de linguagem, verificaram uma média de limiar de detecção de gap desses indivíduos dentro do padrão normativo. Contudo, discorda com estudo realizado por Muniz et al. (2007) que concluíram que os indivíduos com alterações fonológicas podem apresentar alterações de processamento temporal, necessitando de mais tempo para detectar intervalos de tempo entre estímulos auditivos em relação às crianças sem desvio fonológico ${ }^{17}$.

A média de limiares de gap dos indivíduos estudados foi correspondente a $15 \mathrm{~ms}$. Esse valor é acima da média de 9,5 ms encontrada por Barreto, Muniz e Teixeira ${ }^{13}$, em crianças da mesma faixa etária, porém sem desvio fonológico. Apesar do padrão de normalidade de detecção de gap, os indivíduos estudados necessitam de maior intervalo de tempo para perceber diferenças entre os sons, em relação aos indivíduos que não possuem desvio fonológico.

$\mathrm{Na}$ habilidade de ordenação temporal de frequências, na amostra estudada, observou-se que a maior parte da amostra obteve padrão normativo de acordo com o estabelecido pela literatura. Sendo que das 12 crianças avaliadas, foi necessária aplicação do teste na modalidade imitando em três delas, verificando-se melhor desempenho na solicitação de resposta não-verbal em relação à verbal. A dificuldade na modalidade nomeando, pode ser explicada pela necessidade de integração inter-hemisférica (via corpo caloso) dos estímulos na solicitação de resposta verbal, o que não ocorre na solicitação não-verbal, levando a conclusão de que esses indivíduos pudessem apresentar uma dificuldade na integração auditiva ${ }^{18}$. Pode-se verificar, também, que apenas um indivíduo apresentou alteração na solicitação de resposta verbal e não-verbal para o teste PPS, sugerindo uma alteração na habilidade de ordenação temporal de frequências. Um estudo realizado por Balen (2001) com indivíduos de 7 a 11 anos sem alterações de linguagem verificou que o desempenho das crianças no Teste de Padrão de Frequências na modalidade de resposta não-verbal foi significativamente melhor do que na solicitação de respostas verbais, corroborando os achados do presente estudo ${ }^{19}$. Acredita-se que o ocorrido possa ser atribuído à necessidade de integração inter-hemisférica dos estímulos na solicitação de resposta verbal, o que não ocorre na solicitação não-verbal.

$\mathrm{Na}$ análise das variáveis, grau de severidade do desvio fonológico, e desempenho dos indivíduos no teste de ordenação temporal de frequências, observa-se uma relação em que, quanto maior o grau de severidade, pior o desempenho do indivíduo no teste PPS (apesar da normalidade obtida por maior parte da amostra). Tais achados demonstram influência entre a severidade do desvio fonológico e o desempenho dos indivíduos no teste de ordenação de frequências. Atribui-se o encontrado à aquisição adequada do sistema fonológico depender, não apenas da consciência de quais sons compõem a língua, mas, também, da organização e manipulação desses sons na formação das palavras a serem ditas.

\section{CONCLUSÃO}

A maior parte das crianças com desvio fonológico estudadas apresentou integridade das habilidades de ordenação e resolução temporal. Contudo, a análise da habilidade de resolução temporal demonstrou que elas necessitam de maior intervalo de tempo para perceber diferenças entre os sons, quando comparadas às crianças que não possuem desvio fonológico.

As crianças com desvio fonológico participantes do estudo parecem apresentar dificuldades na organização fonológica e sequencialização adequada dos sons, o que pode supostamente ter refletido no seu desempenho, considerando a ordenação temporal.

Os resultados do estudo salientam importância da estimulação do processamento temporal nas terapias fonológicas, principalmente nas crianças com desvio fonológico. 


\section{ABSTRACT}

Purpose: to check children's performance in temporal resolution and ordering tests under the presence of phonological disorders. Methods: the sample was made up of 12 people of both genders with ages between 5 and 13 years, diagnosed with phonological deviation. They were submitted to anamnesis, meatoscopy, audiometric tests, Percentage of Correct Consonants (PCC), the Random Gap Detection Test (RGDT) and the Pitch Pattern Sequence (PPS). Results: in relation to the degree of severity, it was verified that $67 \%$ of children had medium phonological deviation, and $33 \%$ mediummoderate. In relation to RGDT and PPS tests, there were observed normal values, according to the normative standards. Comparing the performance in PPS test and the degree of severity, we observed that the higher the severity is, the worse was the performance. The same does not stand for the RGDT. Conclusions: these results showed that children with phonological deviation should have an adequate performance in temporal processing tests. There was a significant relation between the severity of phonological deviance and the performance in PPS test in our sample. These results demonstrated that the influence of the severity of phonological deviance should be related to the ability of temporal ordering.

KEYWORDS: Speech; Auditory Perception; Hearing

\section{REFERÊNCIAS}

1. Mota HB.Terapia fonoaudiológica para os desvios fonológicos. Rio de Janeiro: Revinter; 2001.

2. Linassi LZ, Keske-Soares M, Mota HB. Habilidades de memória de trabalho e o grau de severidade do desvio fonológico. Pró-Fono. 2005; 17(3):383-92-60.

3.Vieira MG, Mota HB, Keske-Soares M. Relação entre idade, grau de severidade do desvio fonológico e consciência fonológica. Rev Soc Bras Fonoaudiol. 2004; 9(3):144-50.

4. American Speech-Language-Hearing Association. Central auditory processing: current status of research and implications for clinical practice. Am J Audiol. 1996; 5(2):41-52.

5. Koslowski L, Wiemes GMR, Magni C, Silva ALG. A efetividade do treinamento auditivo na desordem do processamento auditivo central: estudo de caso. Rev. Bras. Otorrinolaringol. 2004; 70(3): 427-32.

6. Frota S, Pereira LD. Processos temporais em crianças com déficit de consciência fonológica. Rev Iberoam Educac. 2004; 33(9):1-9.

7. Shin JB. Temporal processing: the basics. Hear J. 2003; 56(7):52.

8. Samelli AG, Schochat E. The gaps-in-noise test: gap detection thresholds in normal-hearing young adults. Int J Audiol. 2008; 47(5):238-45.

9. Zaidan E, Garcia AP, Tedesco MLF, Baran JA. Desempenho de adultos jovens normais em dois testes de resolução temporal. Pró-Fono. 2007; 20(1):19-24.
10. Fortes AB, Pereira LD, Azevedo MF. Resolução temporal: análise em pré-escolares nascidos a termo e pré-termo. Pró-Fono. 2007; 19(1):87-96.

11. Garcia VL, Campos DBKP, Padovani CR. Associação entre a avaliação de habilidades de consciência fonológica e processamento auditivo em crianças com e sem distúrbio de aprendizagem. Fono Atual. 2005; 8(31):4-11.

12. Northen JL, Downs MP. Audição na infância. 5. ed. Rio de Janeiro: Guanabara Koogan; 2005.

13. Shriberg LD, Kwiatkowski J. Phonological disorders I: a diagnostic classification system. J Speech Hear Disord. 1982; 47(3):226-41.

14. Keith RW. Random gap detection test. Auditec of Saint Louis; 2000.

15. Barreto MAS, Muniz LF, Teixeira FC. Desempenho da habilidade a resolução temporal em crianças de 07 a 13 anos. Rev Soc Bras Fonoaudiol. 2004; 9(4):220-8.

16. Feniman MR, Keith RW, Cunningham RF. Assessment of auditory processing in children with attention deficit hyperactivity disorder and languagebased learning impairments. Dist Comun. 1999; 11(1):9-27.

17. Muniz LF, Roazzi A, Schochat E, Teixeira CF, Lucena JA. Avaliação da habilidade de resolução temporal, com uso do tom puro, em crianças com e sem desvio fonológico. Rev. CEFAC. 2007; 9(4):550-62. http://dx.doi.org/10. 1590/S1516-18462007000400016 
18. Musiek F, Chemark G. Handbook of (central) auditory processing disorder: auditory neuroscience and diagnosis. San Diego: Plural Publishing Group; 2007. p.231-57.
19. Balen SA. Reconhecimento de padrões auditivos de frequência e de duração: desempenho de crianças escolares de 7 a 11 anos [dissertação]. São Paulo (SP): Universidade de São Paulo; 2001.

RECEBIDO EM: 17/07/2009

ACEITO EM: 13/11/2009

Endereço para correspondência:

Júlia Larissa Ferreira dos Santos

Rua Minas Gerais, 812

Biquinhas - MG

CEP: 35621-000

E-mail: julialarissa@gmail.com

Rev. CEFAC, São Paulo 